\title{
Netherton syndrome: a neonatal case with respiratory insufficiency
}

\author{
Emel Okulu, M.D. ${ }^{a}$, Gaffari Tunc, M.D. ${ }^{a}$, Omer Erdeve, Prof ${ }^{a}$, Yelda Mumcu, M.D. ${ }^{b}$, Begum Atasay, Profa, \\ Erdal Ince, Profc and Saadet Arsan, Profa
}

\begin{abstract}
Netherton syndrome (NS) is a rare, autosomal recessive disease characterized with congenital ichthyosiform erythroderma, hair abnormality and atopic manifestations. This syndrome is caused by recessive mutation in the SPINK5 gene. Disease manifestations vary considerably among NS individuals. We report a newborn presented with severe respiratory insufficiency, hypothermia and erythroderma, was diagnosed as having NS and confirmed with molecular genetic testing. Key words: Infant newborn, Netherton syndrome, respiratory insufficiency.
\end{abstract}

http:/ / dx.doi.org/10.5546/ aap.2018.eng.e609

To cite: Okulu E, Tunc G, Erdeve O, et al. Netherton syndrome: a neonatal case with respiratory insufficiency. Arch Argent Pediatr 2018;116(4):e609-e611.

\section{INTRODUCTION}

Netherton syndrome (NS) is a rare, severe, autosomal recessive disease characterized with congenital ichthyosiform erythroderma, hair abnormality known as 'trichorrhexis invaginata', and atopic manifestations. Recent reports have shown that the gen SPINK5 encodes lymphoepithelial Kazal-type-related inhibitor (LEKTI), a protein localized in the granular and uppermost spinous layers of epidermis as well as in the most differentiated layers of all stratified

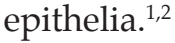

a. Department of Pediatrics, Division of Neonatology, Ankara University, Faculty of Medicine, Ankara, Turkey.

b. Neonatal Intensive Care Unit, Ankara TOBB ETU Hospital, Ankara, Turkey.

c. Department of Pediatrics, Division of Pediatric Infectious Diseases, Ankara University, Faculty of Medicine, Ankara, Turkey.

E-mail address:

Emel Okulu, M.D.: emelokulu@gmail.com

Funding: None.

Conflict of interest: None.

Received: 9-21-2017

Accepted: 1-31-2018
We herein report a newborn NS patient with generalized erythroderma and hypothermia who required invasive respiratory support and was referred for extracorporeal membrane oxygenation (ECMO).

\section{CASE REPORT}

A $3300 \mathrm{~g}$ male newborn was born at 39 weeks' $^{\prime}$ gestation by repeat cesarean section with Apgar scores 6 and 8 at $1^{\prime}$ and $5^{\prime}$ minutes, respectively. He was the second child of nonconsanguineous parents after an uncomplicated pregnancy. He received positive pressure ventilation at delivery room. No meconium was noted at the time of delivery. The infant developed respiratory distress which worsened in following minutes and was intubated. Surfactant was administered twice for the ground-glass appearance at chest radiograph to improve oxygenation. The serial blood gas analysis demonstrated persistent hypoxemia and the infant was referred to our neonatal intensive care unit (NICU) to be evaluated for ECMO support.

On admission to our NICU, the infant had severe hypothermia (body temperature: $32{ }^{\circ} \mathrm{C}$ ) and bradycardia in addition to respiratory failure. Fine and fragile scalp hair, eyebrows and eyelashes, generalized psoriasiform type erythroderma on whole body were recorded (Figure 1). Laboratory findings except hypoxemia were not specific on admission. Echocardiography demonstrated mild pulmonary hypertension without structural abnormality. Light microscopic examination of scalp hair was remarkable for the characteristic 'trichorrhexis invaginata' consistent with the clinical diagnosis of NS (Figure 2), and molecular genetic testing resulted in a homozygous mutation IVS17-1G>A (c.1608$1 \mathrm{G}>\mathrm{A}$ ) of the SPINK5 gene.

The infant could be kept normothermic and was extubated on postnatal $5^{\text {th }}$ day. He developed hypernatremia (165 mEq/L, N:133-145 mEq/L) on the $2^{\text {nd }}$ day which resolved with intravenous fluid therapy. The exfoliative erythroderma has improved with continued use of topical aquaphor. He was treated with bronchodilators 
due to bronchiolitis symptoms on follow-up and was discharged on postnatal $25^{\text {th }}$ day with only topical skin care.

The infant is 5 -months age at the present. He was admitted to hospital with lower respiratory tract viral infection and treated with bronchodilators. He still has generalized erythroderma with desquamation managed with topical care. He is in the follow-up program of the Department of Pediatric Immunology. Genetic counseling was given and prenatal genetic diagnosis was suggested to family for next pregnancy.

\section{DISCUSSION}

Netherton syndrome, is a rare autosomal recessive disease with skin and hair abnormalities. Incidence has been estimated at 1 in 200,000, and it could account up to $18 \%$ of congenital erythodermas. ${ }^{3}$ The patient we report here manifested the typical features of NS as erythroderma, bamboo hair, respiratory symptoms and hypernatremia with SPINK5 gene mutation.

FIGURE 1. The patient with fine scalp hair and erythroderma

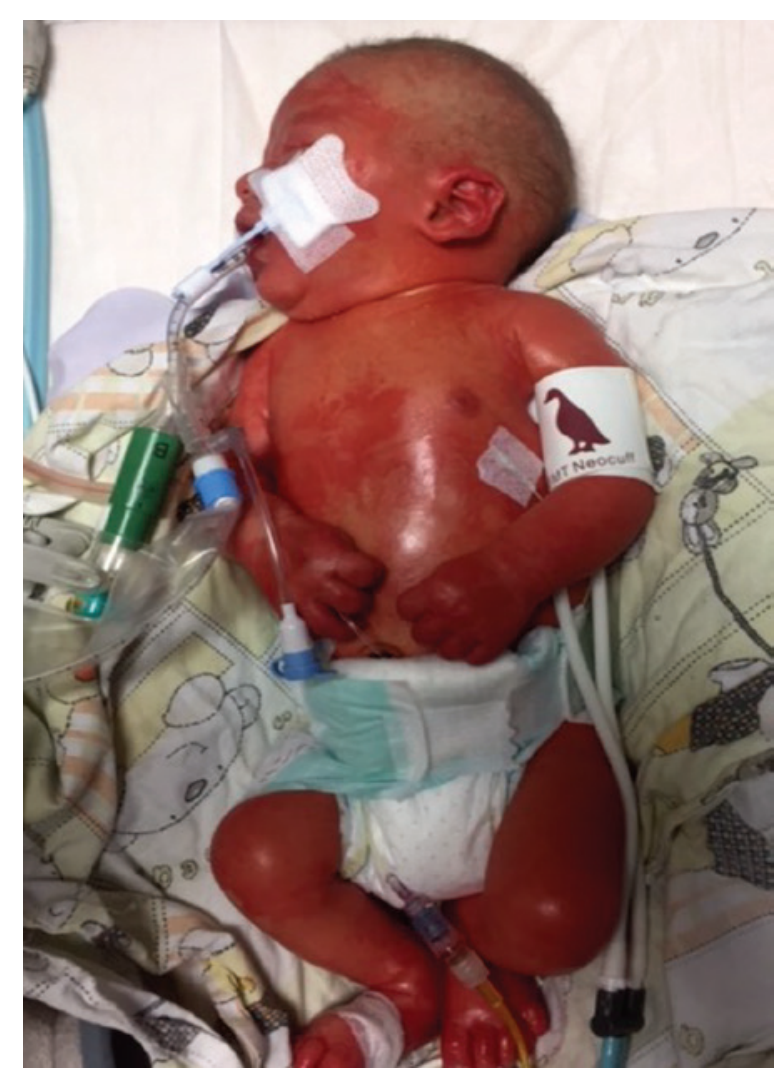

Macknet et al. reported an infant with NS who received ECMO treatment for persistent pulmonary hypertension that was speculated to be secondary to bronchopneumonia due to thick amniotic fluid containing exfoliated epidermal cells. ${ }^{4}$ Our patient was intubated, received surfactant shortly after birth, and referred to our unit for ECMO treatment. Due to mild pulmonary hypertension the infant was treated with conventional therapies. We thought that aspiration of the amniotic fluid containing desquamated epidermal cells caused pneumonia and respiratory insufficiency.

The gene for Netherton syndrome has been identified on chromosome 5q32, named SPINK5 (serine protease inhibitor, Kazal type-5), and encodes an inhibitor of serine proteases called LEKTI. ${ }^{2,5}$ Chavanas et al. have reported the first mutation of SPINK $5,{ }^{5}$ and today 80 different mutations in exonic and intronic regions were reported. ${ }^{6}$ Our patient was homozygous for the intron 17 splice site mutation $(1608-1 G \rightarrow A)$. This mutation was previously defined in an 8 -years old French patient with erythroderma at birth, trichorrhexis invaginata, atopic manifestations, failure to thrive, recurrent infections and enteropathy. ${ }^{7}$

Currently, there is no proven cure or consensus on treatments. Treatment of the skin lesions consists of emollients, antihistamines, antibiotics and topical corticosteroids. ${ }^{6}$ The successful treatment with monoclonal antibody therapies of adult patients with NS have been reported. ${ }^{8,9}$ Our patient has been treated only with emollients and topical antibacterial.

FIGURE 2. Trichorrhexis invaginata at light microscopy of the patient's scalp hair

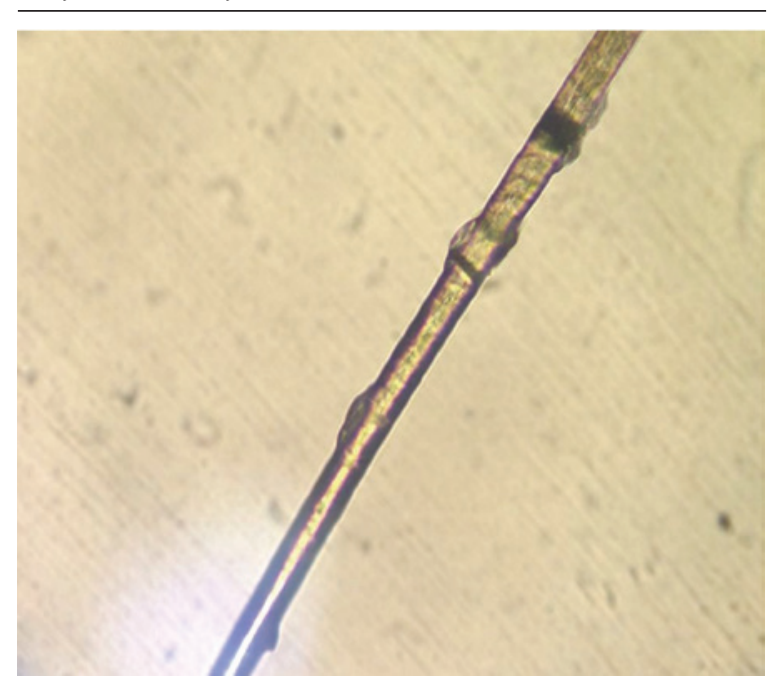


In conclusion, our patient is one of the rare cases presented with respiratory failure at birth and exhibited characteristic findings of NS as skin and hair abnormalities confirmed with SPINK5 gene mutation. Making the diagnosis of Netherton syndrome is challenging in a neonate. The disease has a wide variety of clinical phenotypes. Early diagnosis is essential because of the association of the disease with life threatening complications. It is crucial for prenatal diagnosis in cases of a family history of NS and when consanguinity is implied.

\section{REFERENCES}

1. Bitoun E, Micheloni A, Lamant L, et al. LEKTI proteolytic processing in human primary keratinocytes, tissue distribution and defective expression in Netherton syndrome. Hum Mol Genet. 2003;12(19):2417-30.

2. Chavanas S, Bodemer C, Rochat A, et al. Mutations in SPINK5, encoding a serine protease inhibitor, cause Netherton syndrome. Nat Genet. 2000;25(2):141-2.
3. Pruszkowski A, Bodemer C, Fraitag S, et al. Neonatal and infantile erythrodermas: a retrospective study of 51 patients. Arch Dermatol. 2000;136(7):875-80.

4. Macknet CA, Morkos A, Job L, et al. An infant with Netherton syndrome and persistent pulmonary hypertension requiring extracorporeal membrane oxygenation. Pediatr Dermatol. 2008;25(3):368-72.

5. Chavanas S, Garner C, Bodemer C, et al. Localization of the Netherton syndrome gene to chromosome 5q32, by linkage analysis and homozygosity mapping. Am J Hum Genet. 2000;66(3):914-21.

6. Sarri CA, Roussaki-Schulze A, Vasilopoulos Y, et al. Netherton syndrome: a genotype-phenotype review. Mol Diagn Ther. 2017;21(2):137-52.

7. Bitoun E, Chavanas S, Irvine AD, et al. Netherton syndrome: disease expression and spectrum of SPINK5 mutations in 21 families. J Invest Dermatol. 2002;118(2):352-61.

8. Yalcin AD. A case of netherton syndrome: successful treatment with omalizumab and pulse prednisolone and its effects on cytokines and immunoglobulin levels. Immunopharmacol Immunotoxicol. 2016;38(2):162-6.

9. Roda A, Mendonça-Senches M, Travassos AR, et al. Infliximab therapy for Netherton syndrome. A case report. JAAD Case Rep. 2017;3(6):550-2. 